\title{
Simulación numérica del proceso de mezcla en el convertidor de acero. Efecto del método de carga de los aditivos ${ }^{(\bullet)}$
}

\author{
M. Díaz ${ }^{(*)}$ y M.E. García ${ }^{(* *)}$
}

\begin{abstract}
Resumen Debido a que los tiempos de mezcla y de reacción son muy similares en los procesos que tienen lugar en el interior de un convertidor de acero, es importante el estudio de los tiempos de mezcla en el interior del tanque. Dadas las altas temperaturas, el estudio directo se hace difícil, siendo útiles los modelos matemáticos que aproximen el problema. En este trabajo se propone un modelo matemático sencillo basado en Redes de Zonas, que necesita relativamente pocos medios para la resolución del problema de la mezcla. Finalmente, se analizan las diferencias que se producen en la mezcla según las diferentes formas de carga de los aditivos.
\end{abstract}

Palabras clave: Convertidor. Mezcla. Modelo matemático. Forma de carga.

\section{Numeric simulation of the mixing process inside the steel converter. Effect of the additions charging method}

\begin{abstract}
Due to the similarity of the mixing and reaction times in the processes inside a steel converter, it is important to study the mixing times in the vessel. Owing to the high temperature, the direct determination is difficult, and mathematical models that approach the problem are very useful. In the present work, a simple mathematical model based on Networks of Zones that do not need too much mathematical apparatus for the solution of the mixing problem is proposed. Finally, the different behaviour of the mixing as a function of the additions charging method is analysed.
\end{abstract}

Keywords: Steel converter. Mixing. Mathematical model. Charging method.

\section{INTRODUCCIÓN}

En el proceso LBE se utiliza oxígeno puro para refinar el arrabio y obtener acero. Tras la carga, el convertidor se gira a la posición vertical, utilizada en el proceso de soplado. Se baja la lanza y comienza el soplado de oxígeno. El chorro de oxígeno a alta velocidad inyectado a través de la boquilla de la lanza penetra en la escoria y el metal agitando el baño. Simultáneamente, gases inertes, argón o nitrógeno se inyectan a través de la base del convertidor. La inyección de estos gases mejora las condi-

$(\bullet$ Trabajo recibido el día 25 de julio de 1995.

(*) Dpto. de Ingeniería Química. Facultad de Química. Universidad de Oviedo. C/ Julián Clavería. 33071-Oviedo (España).

(**) Dpto. de Matemáticas. Facultad de Ciencias. Universidad de Oviedo. C/ Calvo Sotelo, s/n. 33071-Oviedo (España). ciones de mezcla y por ello también las de la reacción. Los dos soplados, superior e inferior, provocan el flujo del fluido en el convertidor, forzándolo a circular del fondo a la superficie en el centro y en dirección contraria cerca de las paredes del reactor.

La reacción entre el oxígeno y el metal se localiza al principio en pequeñas zonas, pero se extiende rápidamente al aumentar la concentración de gas CO. La agitación y la circulación del baño provocan una renovación del metal en la interfase metalescoria que contribuye de forma substancial a las altas velocidades de refino. La reacción de oxidación del silicio que se inicia en los primeros momentos, es fuertemente exotérmica, elevando la temperatura del baño y, por lo tanto, permitiendo la adición de chatarra a la carga para consumir el exceso del calor disponible (1). Se oxidan también otras impurezas del baño como el carbono, el manganeso y el fósforo. 
Los estudios de problemas de mezcla en la bibliografía, se han centrado principalmente en el estudio de tanques cilíndricos agitados mecánicamente por turbinas ( 2 y 3 ). Sin embargo, la mezcla es un factor decisivo en procesos metalúrgicos y siderúrgicos donde la temperatura del baño no permite la agitación mecánica. El método de agitación suele ser la inyección de gases, ya sean inertes, cuando su objeto sea únicamente agitar, o reactivos, cumpliendo entonces una doble función de elemento agitador y participante en la reacción (4 y 5). En el caso del proceso LBE, como se ha dicho, tienen lugar simultáneamente ambos mecanismos.

El estudio de los procesos de mezcla aquí indicados se ha realizado con cierta frecuencia en modelos fríos que utilizan habitualmente agua (6). Existen, asimismo, estudios de modelización matemática por diversos métodos; así, por ejemplo, mediante el modelo de turbulencia $\kappa-\epsilon$ se han obtenido velocidades en una sección de una cuchara durante el proceso de refino (7 y 8).

En este trabajo, se estudia el caso concreto del proceso de mezcla en un convertidor LBE de $250 \mathrm{t}$ de forma cilíndrica y fondo troncocónico. En el control de las reacciones, en que el tiempo de mezcla y la velocidad de reacción son parecidas, como en este caso, es esencial conocer los mecanismos de la mezcla (9). El objeto es mejorar los conocimientos sobre los procesos de mezcla que acontecen en el interior del convertidor de acero durante el soplado. Para ello, mediante el modelo desarrollado se considera la evolución de la concentración de una traza en los distintos puntos del convertidor. A partir de dicha concentración se puede obtener el tiempo de mezcla que, por las razones ya dichas, es importante en el tipo de reacciones que tienen lugar en el interior del convertidor y por ello en la economía del proceso. Finalmente, se estudia la influencia de la forma de la carga y del soplado en la mezcla, lo que puede servir de ayuda en la mejora de la operación.

\section{ANÁLISIS DEL SISTEMA}

\subsection{Descripción del proceso}

Desde el punto de vista del soplado inferior, se pueden considerar dos períodos en la operación (10):

\subsubsection{Período inicial}

Se extiende desde el principio del soplado del oxígeno hasta dos minutos antes del final. El control se realiza variando el flujo de oxígeno y la altura de la lanza sobre el baño. Se pueden considerar tres subperíodos.

\subsubsection{Ignición}

Durante los primeros segundos se produce una intensa reacción con la carga. Los fundentes se añaden a la carga, inmediatamente después de la ignición, para proteger el recubrimiento del horno y producir en esta fase temprana del soplado suficiente escoria básica para desulfurar y desfosforizar la carga y reducir estas impurezas a niveles finales aceptables. Durante los primeros minutos de soplado y para acelerar y mejorar la homogeneización del baño, se inyectan 55,6 L/s

\subsubsection{Desilicación}

Tras la ignición, el silicio contenido en el baño se oxida rápidamente. Lo mismo sucede con parte del hierro y del manganeso. Estos óxidos pasan a la escoria. Cuando la cantidad de $\mathrm{SiO}_{2}$ en la escoria aumenta, esta se hace más viscosa y a los gases les cuesta más pasar a través de ella. Para evitar que se derrame es necesario reducir el flujo de oxígeno y parar el flujo de gases inertes.

\subsubsection{Descarburación}

Debido a la inyección de gases por el fondo, la presión parcial del CO desciende y la velocidad de descarburación se estabiliza. Como la presión parcial del CO en el proceso LBE es menor que en el $\mathrm{LD}$, el desprendimiento de $\mathrm{CO}$ en el LBE es mayor que en el LD. Cuando se añade el mineral refrigerante, la descarburación aumenta debido al incremento de FeO en el baño. Para evitar el derrame del baño, debe reducirse el flujo de oxígeno.

\subsubsection{Periodo final}

Durante los últimos minutos de soplado de oxígeno, se inyectan gases inertes a través del fondo para buscar un incremento del manganeso residual y una reducción del $\mathrm{FeO}$ en la escoria.

La distribución de los aditivos y su mezcla con la carga es fundamental para las reacciones que tienen lugar. Del tiempo que tarden en mezclarse dependerá la eficacia de las mismas. En los siguientes apartados se desarrolla un modelo matemático que permite estudiar la dispersión de un aditivo en el baño, su distribución y los tiempos de mezcla dependiendo del soplado y de la forma de la carga.

\subsection{Modelo fluidodinámico}

En la figura 1 se observa una sección de un convertidor. La altura total es de aproximadamente 10 


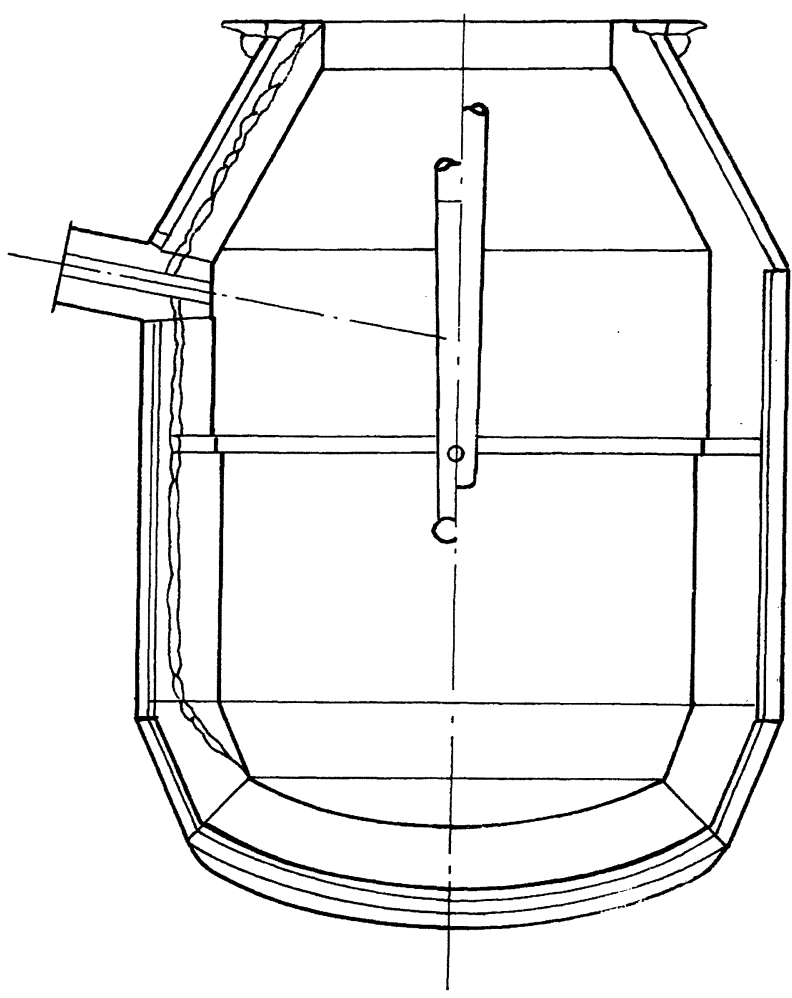

FIG. 1.- Geometría del convertidor de acero.

\section{FIG. 1.- Steel converter geometry.}

m. La carga se sitúa en el fondo del convertidor. En la figura 2 se representa una sección axial de la carga ya dividida en elementos para aplicar el modelo.

Estos elementos tienen simetría axial e igual volumen. Son anillos de sección rectangular con centro en el eje de simetría del convertidor. Como el radio de estos anillos es distinto y sus volúmenes son iguales, su sección axial tendrá distinta área, siendo esta tanto menor cuanto mayor es su radio, o distancia del eje. El suponer simetría axial lleva a que dentro de cada uno de estos elementos anulares

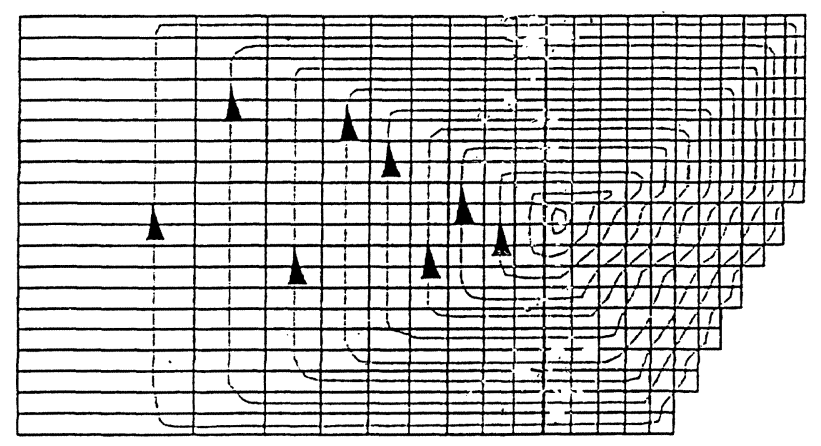

FIG. 2.- Celdas y líneas de flujo usadas por el modelo matemático.

Fig. 2.- Cells and streamlines used by the mathematical model. existe siempre una mezcla perfecta, es decir, la concentración en un elemento se asume uniforme en cada instante.

De acuerdo con las observaciones experimentales, el líquido circula en el tanque, bajo el efecto del soplado, de forma aproximadamente concéntrica a las paredes, siendo el sentido de circulación de abajo a arriba en la zona del eje y de arriba hacia abajo en la zona de las paredes. Este flujo se simulará superponiendo a los elementos propuestos líneas de flujo que transcurran paralelas a los bordes de la sección (Fig. 2). Se comienza por las hileras exteriores. Debido a que la forma de la sección no es rectangular, este modo de proceder tiene como consecuencia que las líneas situadas más al centro o próximas al vórtice de la sección axial están condicionadas por las exteriores, al atenerse al criterio de hacer pasar por cada casilla o elemento una única línea. La situación del vórtice o centro de las líneas de flujo también vendrá condicionada por esta forma de proceder.

Estas líneas de flujo marcarán la dirección del mismo en cada elemento, es decir, por el lado que entra y por el que sale. Se supondrá que existe, con las casillas de flujo adyacentes, un intercambio de fluido debido a la turbulencia, y que este intercambio es proporcional, con factor de proporcionalidad $\beta$, al flujo que atraviesa cada elemento, $q$. Estas interacciones entre celdas se reflejan en la figura 3.

La ecuación a aplicar a cada celda será:

$v_{i j} \frac{\mathrm{d} c_{\mathrm{ij}}}{d \mathrm{t}}=q(\overbrace{c_{\mathrm{ij}-1}-c^{\mathrm{ij}}}^{\text {flujo }}+\beta(\overbrace{c_{\mathrm{i}}-1_{\mathrm{j}}+\mathrm{c}_{\mathrm{i}+1 \mathrm{j}}-2 \beta c_{\mathrm{ij}}}^{\text {a turbulencias }})$

Esta ecuación (11 y 12) contiene los parámetros $\beta$ y $q$, siendo las incógnitas las concentraciones de la traza en el tiempo en cada casilla. Hay que tener en cuenta que las casillas situadas en las esquinas de las líneas de flujo y en el chaflán de la sección tendrán un comportamiento algo diferente. Así, se ha tomado como criterio a aplicar, a la hora de considerar intercambios, que una casilla intercambia materia con las casillas que comparte lado, pero con la cual no existe un flujo directo, $q$. En el chaflán es necesario suponer que el flujo sigue una dirección diagonal, pasando a través de los vértices y no de los lados de la casilla. Teniendo en cuenta estos casos particulares, se plantea una ecuación para cada elemento. Así, se tendrá un sistema de ecuaciones diferenciales lineales de primer orden con un número de ecuaciones igual al número de elementos. El proceso de escritura de las ecuaciones se ha sistematizado mediante un programa FORTRAN. Este divide primero la sección axial teniendo en cuenta el número de filas de elementos que se quiera y el número máximo de columnas deseado. 


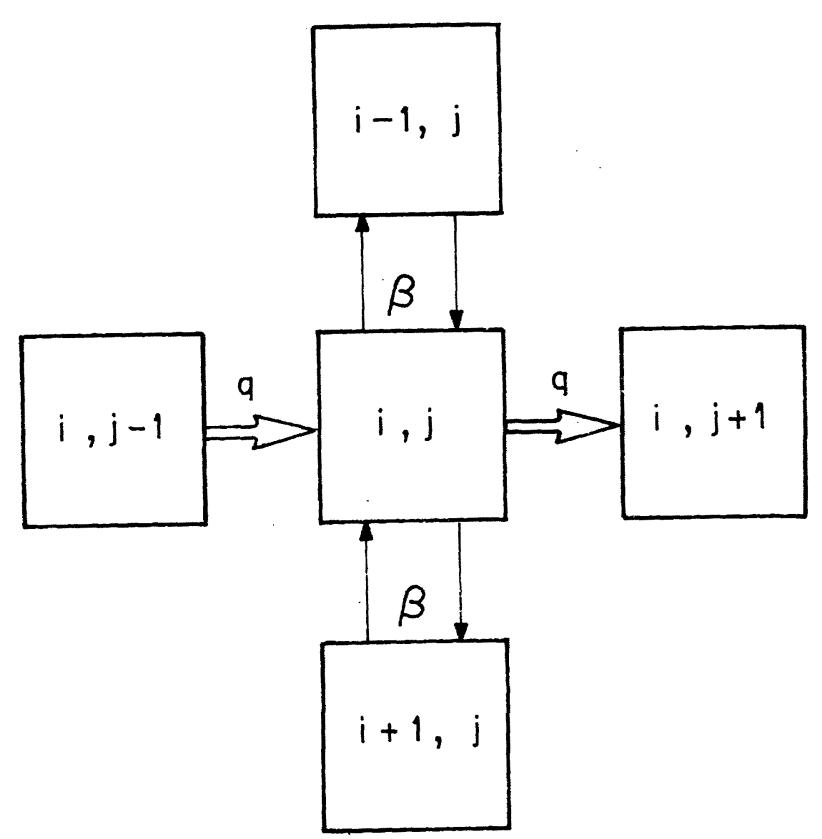

FIG. 3.- Interacciones entre las celdas del modelo.

Fig. 3.-Interactions between the cells in the model.

Debido al chaflán y a que las secciones de los elementos son rectangulares se cometerá un pequeño error en el volumen total, al no considerar parte de la sección axial.

Una vez determinados geométricamente y numerados los elementos, se procede a determinar las conexiones entre ellos debidas al flujo principal. Así, en una matriz se almacenará, para cada elemento, aquel del cual procede el flujo, y en otra, el elemento hacia el cual sale el flujo. Esto se hará atendiendo al criterio de paralelismo del flujo principal con los bordes de la sección; después, se determinarán los elementos que intercambian flujo. Esto se hará tomando los elementos que comparten lado y excluyendo los que están en la misma línea de flujo, almacenados en el proceso previo.

Conocidas estas relaciones, se procede a la escritura de las ecuaciones en un fichero. El conjunto forma un sistema de ecuaciones diferenciales de primer orden. El fichero que las contiene, convenientemente encabezado para que sea una función de FORTRAN, se puede utilizar para resolver el sistema con las librerías IMSL (13) por el método de Runge-Kutta, concretamente con la librería SMATH5A.LIB. La solución del sistema se almacenará en un fichero en el que cada línea contenga la concentración de la traza en una casilla, en un instante dado. Como se ha trabajado con 120 pasos en el tiempo, si $n$ es el número de casillas o elementos, el fichero del resultados tendrá $n \times 120$ líneas que contienen la evolución de la concentración en el tiempo de todos los elementos.

\section{RESULTADOS}

A partir del fichero de resultados, mediante programas FORTRAN que extraigan ciertos tipos de datos y los reordenen, o bien los contrasten con datos experimentales, se pueden obtener y comparar resultados para distintas condiciones, que se obtendrán variando los parámetros.

\subsection{Visualización de la evolución de la concentración}

Tomando la concentración de todos los elementos en tiempos consecutivos, se puede ver la evolución de la mancha sobre la sección axial, desde el momento de la inyección hasta la total homogeneización. Para visualizar intervalos de concentraciones, se ha tomado el rango de concentraciones alcanzadas en escala logarítmica y se ha dividido en intervalos iguales entre el valor máximo y el mínimo. Cada subrango de concentraciones se puede representar de un color. En una representación simplificada en blanco y negro sólo se consideran tres intervalos que toman los tonos negro, gris y blanco.

En el momento de la inyección se ve una mancha negra cerca de la zona de carga que en el modelo se sustituye por la inyección de una traza. Esta mancha se va difuminando, aumentando una zona de concentración media de color gris, al tiempo que toda la mancha es arrastrada por el flujo alrededor de la sección. En el momento de mezcla total todo el tanque presenta el mismo tono gris.

\subsection{Curvas pulso-respuesta}

Si se se extrae del fichero de resultados la evolución de la concentración, a lo largo del tiempo en una casilla, se obtendrán las curvas tiempo-concentración que se ven en la figura 4. Estas curvas son diferentes según la zona; en casillas próximas al borde de la sección, y cerca del punto de inyección presentan un pronunciado pico. Otras curvas sobre la misma corriente, pero más lejos del punto de inyección, presentan picos también, pero se amortiguan al aumentar el recorrido del flujo, puesto que cada vez la mancha llega más diluida. Este efecto de suavización del pico se observa también para los puntos situados en corrientes principales más al interior, desapareciendo incluso en puntos cercanos al vórtice de flujo de la sección. En estos últimos puntos, la concentración ascenderá lentamente hasta la de mezcla total.

Comparando las curvas del modelo con las experimentales de modelos fríos, se observa que las curvas correspondientes a puntos más próximos al centro de la sección, donde la traza llega por 


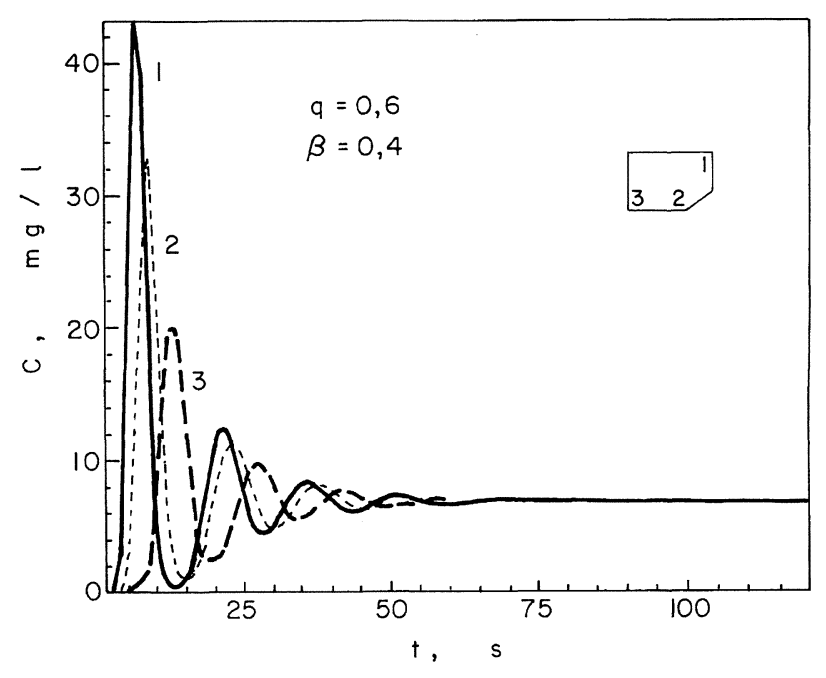

FIG. 4.- Curvas tiempo-concentración obtenidas en tres puntos del convertidor para carga puntual central.

Fig. 4.- Time-concentration curves obtained in three points in the converter for charge in a point in the center of the tank.

difusión debida al flujo turbulento, son las que más se parecen. Ello indica que la naturaleza del flujo en el convertidor es más turbulenta que en los casos de flujos generados por turbinas, donde sucesivos álabes "empujan" y ordenan el flujo. Esta conclusión puede aplicarse en el posible perfeccionamiento de este modelo. La variación de la concentración de la traza en las paredes, donde, según el modelo, esta llega principalmente arrastrada por la corriente, es más oscilante. Estas oscilaciones afectarán en la valoración del tiempo de mezcla.

\subsection{Tiempo de mezcla}

Se define el tiempo de mezcla global como el tiempo transcurrido desde la inyección de la traza hasta que la concentración en cualquier punto del tanque se sitúa en una banda de $\pm 5 \%$ de la concentración de mezcla total. El tiempo de mezcla local se define análogamente, pero referido a la variación de concentración de traza en un punto. Este tiempo de mezcla se puede calcular gráficamente, como se muestra en la figura 5a), trazando dos líneas, una por encima de la concentración de mezcla $c_{\infty}$ a 1,05 y otra por debajo a $0,95 c_{\infty}$. El instante a partir del cual toda la gráfica está incluida dentro de esta banda da el tiempo de mezcla. Esto es más cómodo hacerlo mediante un programa FORTRAN. Suponiendo que se han tomado datos en tiempo suficientes para que alcance la concentración de mezcla, se lee este fichero de datos hasta el final, se almacenan estos datos en un vector y se toma como tiempo de mezcla la última concentración. Luego, leyendo los datos del final al principio, se tomará como tiempo de mezcla aquel que corresponda al tiempo siguiente a la primera concentración que no esté dentro del intervalo 0,95-1,05 $c_{\infty}$. Procediendo de este modo, se calcularía el tiempo de mezcla para distintos valores de los parámetros del modelo $\beta$ y $q$. Las relaciones de estos parámetros con el tiempo de mezcla se pueden ver en la figura $5 b$ ). El tiempo de mezcla desciende al aumentar $q$, es decir, el flujo alrededor del tanque. También desciende al aumen$\operatorname{tar} \beta$, es decir, la difusión debida a la turbulencia.

\section{EFECTO DE LA FORMA DE CARGA DE LOS ADITIVOS}

La carga de los aditivos en un convertidor, se realiza volcando un contenedor con los aditivos sobre el convertidor. El método de carga puede influir sobre el tiempo y la forma de la mezcla $y$,
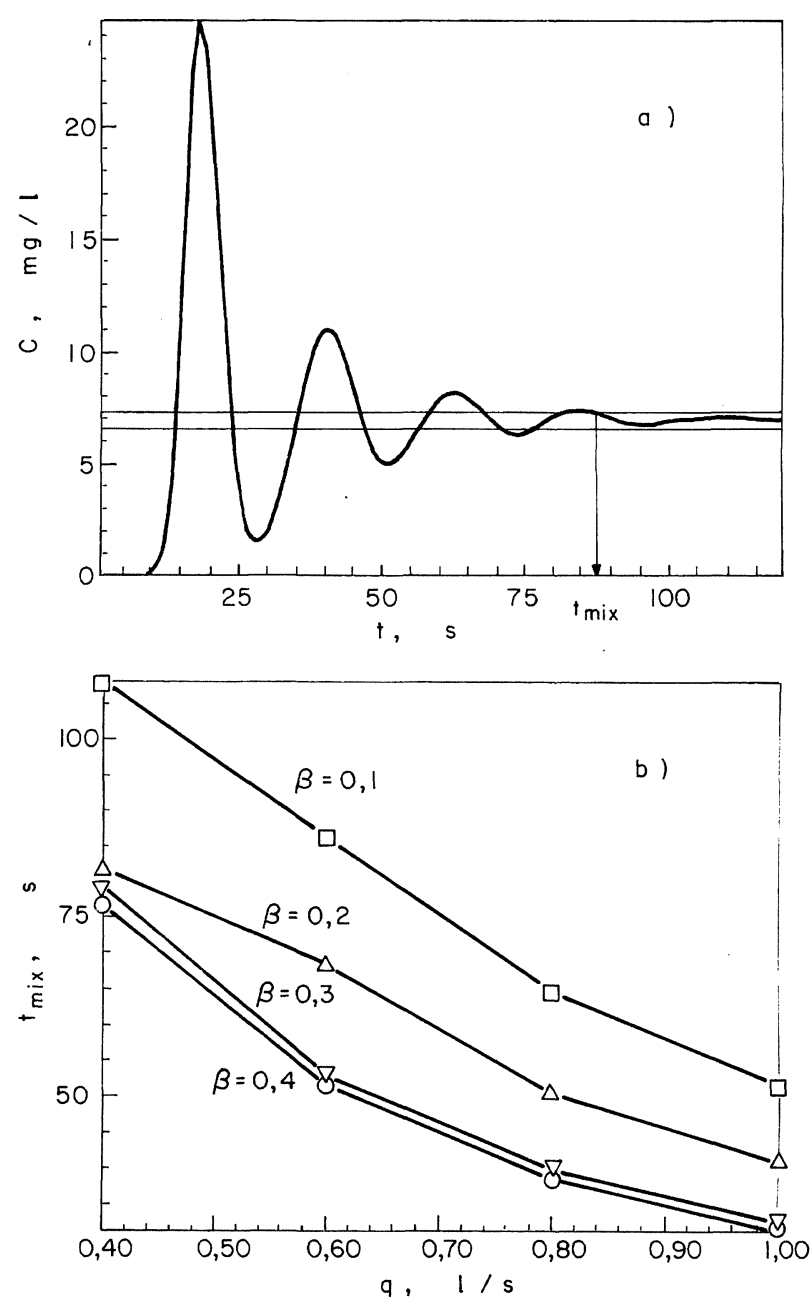

FIG. 5.- Tiempo de mezcla.

a) Modo de obtención. b) Relación con $q$ y $\beta$.

Fig. 5.- Mixing time.

a) Determination. $b$ ) Relation with $q$ and $\beta$. 
por ello, en las reacciones que tienen lugar, lo que hace interesante su estudio para un posible control de la forma de la carga según el efecto que se busque. Se han considerado dos formas ideales de carga (Fig. 6):

a) Carga profunda central. Es la que más se parece a los modos habituales de carga, donde la carga se vierte de golpe sobre una zona aproximadamente central.

b) Carga distribuida superficial. La carga se repartiría en la superficie de todo el convertidor formando una capa de grosor más o menos uniforme sobre toda ella.

De acuerdo con la forma de carga se ha estudiado la evolución de la concentración de la traza, quedando reflejada en la figura 7. Los intervalos de tiempo y los parámetros utilizados para las dos formas de carga son los mismos. Se ve que una carga distribuida favorece que el aditivo alcance antes las paredes y el fondo del convertidor.

Extrayendo la variación de la concentración con el tiempo, se ve que el tiempo de mezcla en las paredes y el fondo va a ser menor en el caso de carga distribuida superficial como se deduce de las

a

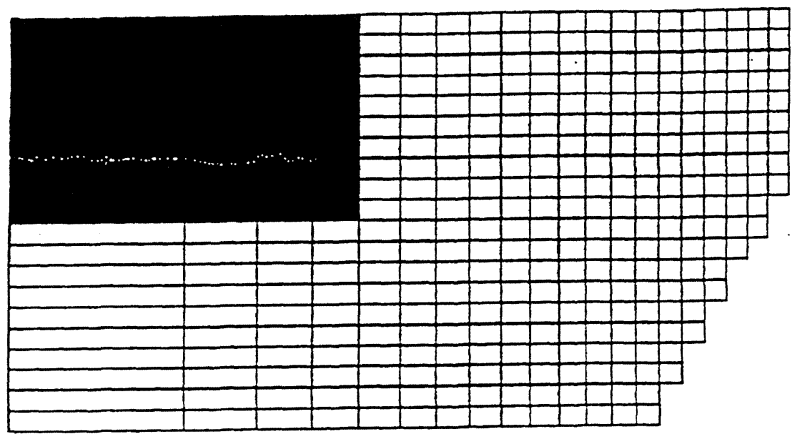

b

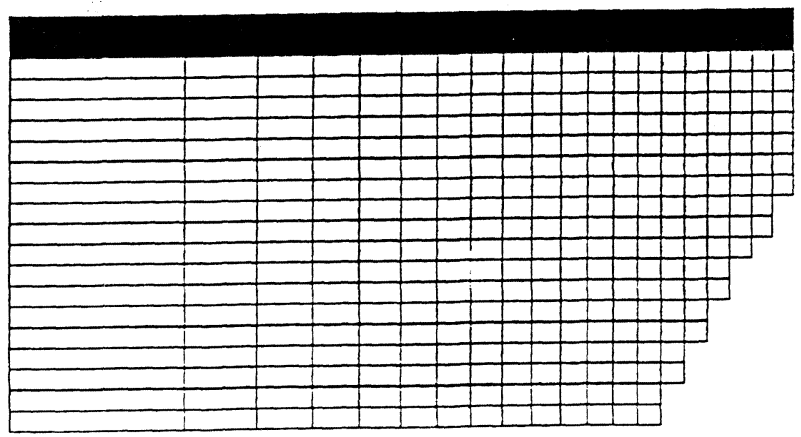

FIG. 6.- Formas de carga estudiadas.

a) Profunda central. b) Distribuida superficial.

Fig. 6.- Studied charging methods.

a) Central and deep. b) Superficial and distributed.
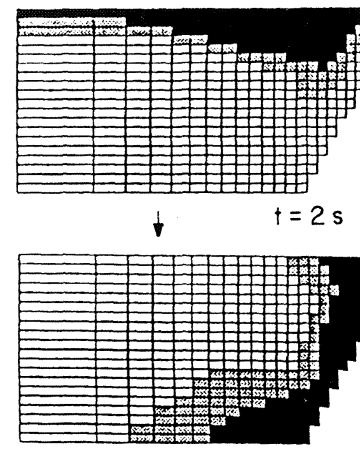

1

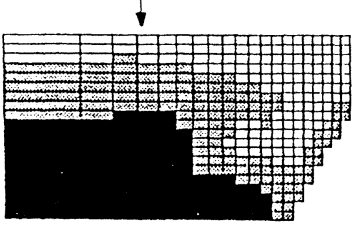

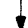

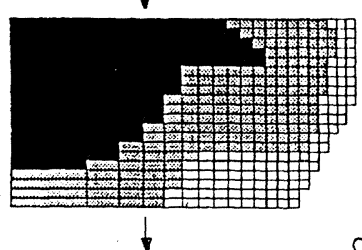

a )
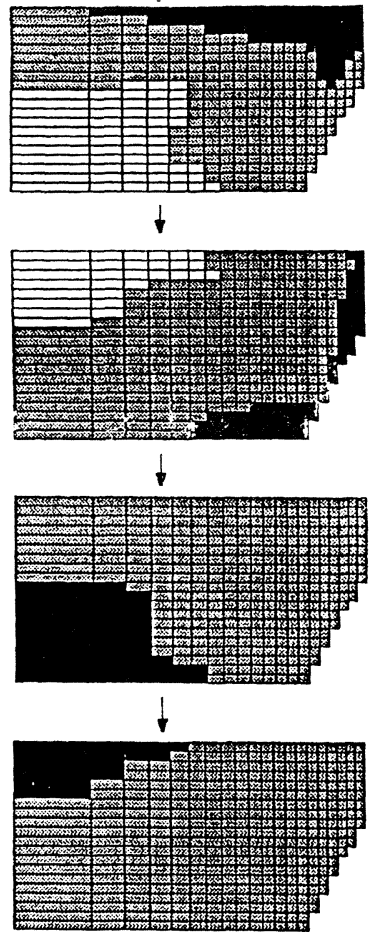

$t=16 \mathrm{~s}$
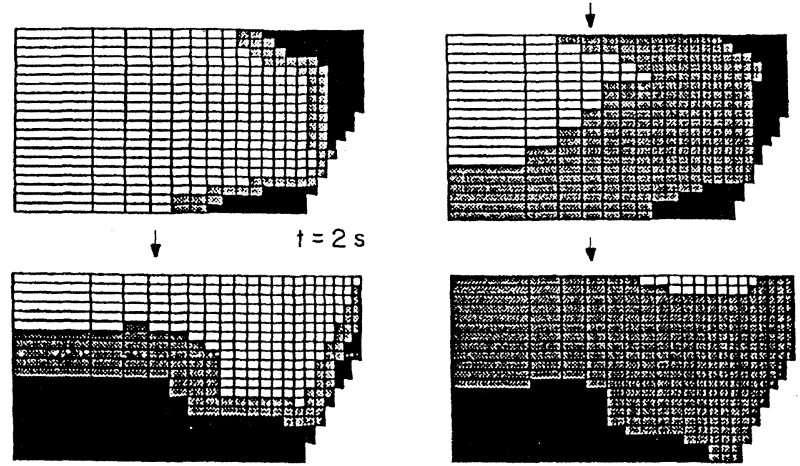

$\checkmark$

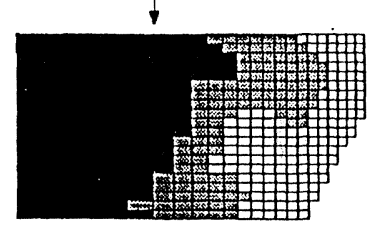

$\downarrow$

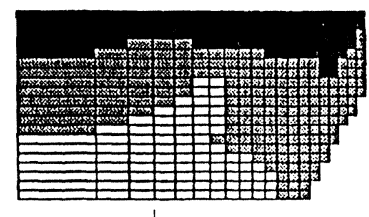

$\downarrow$

b )

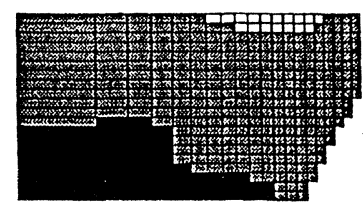

1

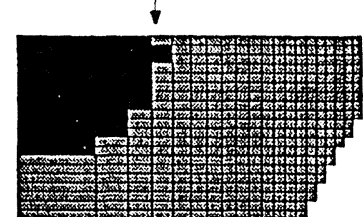

$\downarrow$

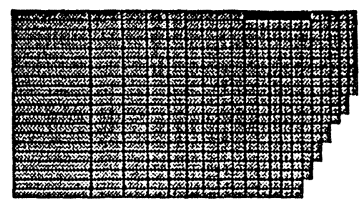

$t=16 \mathrm{~s}$

FIG. 7.- Evolución de la concentración de la traza sobre una sección axial para las formas de carga a y b.

Fig. 7.- Trace concentration evolution on an axial section for the charging methods $a$ and $b$.

curvas tiempo concentración de la figura 8. Así, en el punto 1 del tanque, para la carga profunda central, el tiempo de mezcla es de $172 \mathrm{~s}$, mientras que 

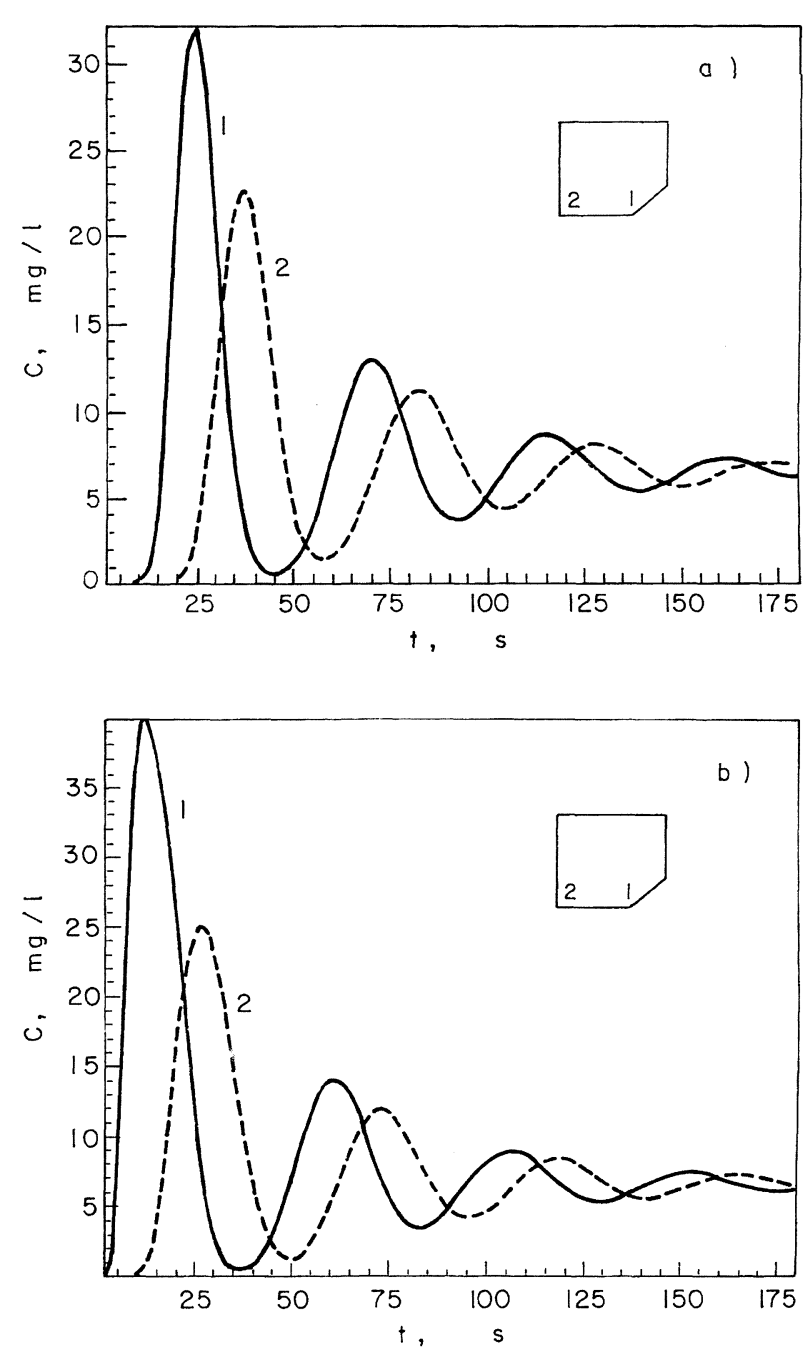

FIG. 8.- Curvas tiempo-concentración para las formas de carga a y $b$.

Fig. 8.- Time-concentration curves for the charging methods $a$ y $b$.

para la carga distribuida superficial es de $162 \mathrm{~s}$. La diferencia del tiempo de mezcla es de, aproximadamente, un $6 \%$ según la forma de carga. La diferencia es la misma en el punto 2 .

\section{CONCLUSIONES}

El modelo matemático descrito proporciona una aproximación al problema de la mezcla en el convertidor de acero. El modelo es sencillo, generando un sistema lineal de ecuaciones diferenciales lineales de primer orden. El modelo funciona bien para el cálculo de los tiempos de mezcla al comparar con resultados experimentales. Cualitativamente, se obtiene una buena respuesta en determinadas zonas, sobre todo en aquellas en que actúa la difusión turbulenta más que el flujo de arrastre directo promovido por el dispositivo de agitación. Ello hace supo- ner que el flujo no es tan ordenado como impresiones de primera vista sugieren. Este modelo proporciona una solución sencilla, que no requiere grandes medios de software y con pocos parámetros, cuya relación intuitiva con parámetros físicos es clara. Se ha estudiado la influencia de la forma de la carga observándose como resultado que la forma teórica de adición se traduce en un mayor o menor tiempo de mezcla en algunas zonas del convertidor.

\section{Símbolos}

$c_{\mathrm{ij}} \quad$ Concentración de la casilla $i j$. Concentration in cell ij.

$c_{\infty} \quad$ Concentración de mezcla total. Concentration when total mixing has been achieved.

n Número de elementos.

Element number.

$q \quad$ Flujo volumétrico para cada tubo de flujo.

Volumetric flow for each loop.

$t$ Tiempo.

Time.

$t_{\text {mix }} \quad$ Tiempo de mezcla.

Mixing time.

$\nu_{\mathrm{ij}} \quad$ Volumen de cada celdilla.

Cell volume.

$\beta \quad$ Parámetro de intercambio.

Interchanging parameter.

$\epsilon \quad$ Potencia de agitación.

Stirring power.

\section{REFERENCIAS}

(1) MacNamara, J., Conway, T.R. y Suhay, W.S. "BOFSteelmaking 1", Ed. Board, Nueva York, 1974: 23.

(2) Kresta, S.M., y Wood, P.E. AIChE J., 37, 3, 1991: 448.

(3) Ranade, V.V., y Bourne, J. R. Chem. Eng. Comm., 99, 1991: 33.

(4) Kato, T. Trans. ISIJ., 26, 1986: 851.

(5) Kishimoto, Y., Takeuchi, S., Fuji, T., Nozaki, T., Take, H., Ueda H. e ImaI, T. Int. Oxygen Steelmaking Cong. Technish wissenschaflicher Verein "Einsenhutte Osterreich". Linz (Austria), 1987: 149.

(6) Woo, J.S., Szekely, J., Castillejos, y Brimacombe, A.H. Met. Trans. B, 213, 1990: 269.

(7) Crevet, J.H. y El Kaddah, N. Int. J. Heat Mass Transfer., 25, 1982: 487.

(8) Tsujino, R., Nakashima, J., Hirai, M. y Sawada, I. ISIJ Int., 29, (7), 1989: 589.

(9) Ou, J.-J. y Ranz, W.E. Chem. Eng. Sci., 38, (7), 1983: 1005.

(10) ENSIDESA. "Formación, Aceros. Tomo 1", mayo 1982.

(11) Mann, R. Chem. Eng. Res. Des., 64, 1986: 23.

(12) Wang, Y. y Mann, R. Trans. I. Chem. E., 70, (A), 1992: 282.

(13) IMSL Library, MATH/LIB, Houston, TX, 1987. 